\title{
ENVEJECIMIENTO \\ Y ENFERMEDAD DE ALZHEIMER
}

\section{M. ${ }^{a}$ Teresa Algado, Angel Basterra y J. Ignacio Garrigós \\ Universidad de Alicante}

\section{EL PROCESO SOCIAL DEL ENVEJECIMIENTO}

El envejecimiento es un proceso dinámico, evolutivo y diferencial, fruto de una gran variedad de variables y en el que se interrelacionan múltiples factores, todos ellos necesarios para analizar y comprender la vejez en su realidad global. De ahí que el fenómeno de la vejez haya sido analizado desde muchas perspectivas y por numerosas disciplinas.

En el análisis de la vejez distinguimos dos procesos profundamente relacionados. Por un lado, el envejecimiento individual o biológico, fenómeno continuo e irreversible que experimentan los individuos en sus organismos a lo largo de las distintas etapas de su ciclo vital; y, por otro, el envejecimiento de la población o demográfico, proceso a través del cual los países desarrollados experimentan un progresivo incremento de la cantidad y proporción de personas mayores de 65 años. En efecto, las reacciones ante los cambios biológicos, fisiológicos o psicológicos que experimentan los individuos dependen del contexto social en el que se producen, de sus distintas experiencias, de sus distintos estilos de vida. En definitiva, la estructura social concreta de cada sociedad influye en los procesos individuales de envejecimiento o en los procesos de envejecimiento de una generación. Pero, por otro lado, el hecho de que cada vez haya más personas en las edades avanzadas repercute en el status de las mismas, en sus papeles sociales, en la interacción entre distintas generaciones y 
afecta a la sociedad en general. Se trata, pues, de dos niveles distintos de un mismo proceso: el proceso social del envejecimiento.

El envejecimiento biológico ha sido tradicionalmente definido de dos modos distintos: por un lado, como un proceso progresivo de deterioro y de destrucción del organismo. Es cierto que a medida que avanza la edad aparecen una serie de lesiones, enfermedades, disfunciones, disminución de capacidades físicas y mentales. Pero no es menos cierto también que este deterioro no afecta a todos los individuos por igual ni con la misma intensidad. También ha sido definido como un conjunto de cambios naturales que, desde el momento del nacimiento y hasta la muerte, se presentan de modo secuencial y afectan a todos los seres vivos. Este tipo de envejecimiento no se debe exclusivamente a factores endógenos, es decir, genéticos o debido a cambios moleculares o celulares, sino también a factores exógenos o medioambientales, como la clase social a la que uno pertenece, el estilo de vida, los recursos económicos, la profesión, el nivel educativo o el tipo de hábitat, por citar algunos de los más relevantes.

Respecto del envejecimiento de la población, no ha habido una plena conciencia, hasta fechas recientes, de que en los países desarrollados, y en particular en Europa, constituía una verdadera revolución —se ha hablado de la revolución gris - cuyas consecuencias afectan, y afectarán todavía más en el futuro, a la organización económica y social de nuestros países tan profundamente como lo han hecho otras grandes revoluciones del pasado. Los cambios demográficos producen sus efectos muy lentamente y a largo plazo, no de forma instantánea, por lo que los países y sus gobiernos no son conscientes de ellos, no llegan a percibirlos, hasta que no afectan a su organización social y plantean problemas que exigen una toma importante de decisiones. Y, sin embargo, esos efectos son a veces de tal envergadura y repercuten de forma tan compleja en el terreno educativo, familiar, laboral, económico, político y asistencial que, en ocasiones, no admiten soluciones a corto plazo e incluso obligan a plantearse cambios drásticos en las políticas de los países afectados.

El aumento, tanto en números absolutos como en porcentajes, de las personas de edad avanzada en el conjunto de la población es un fenómeno que ya había sido previsto tanto por los demógrafos como por los expertos en ciencias sociales. Que cada vez el número de personas que lleguen a edades avanzadas sea mayor supone un logro de la humanidad, un triunfo de la sociedad, y, por lo tanto, debe considerarse como algo positivo. Sin embargo, la opinión pública y los responsables de las políticas sociales y económicas parecen estar sorprendidos por la amplitud de su impacto sobre las estructuras y las tendencias sociales, a la vez que preocupados por lo que consideran un desastre inminente.

Ciertamente, y sin ánimo de ser pesimistas, estamos ante un fenómeno de importantes implicaciones no sólo económicas y sociales, sino también individuales. La evolución demográfica de la estructura por edad afectará a muchos aspectos de la vida social, entre ellos a la familia, al mercado de trabajo, a las 
pautas de jubilación, a la vivienda, al sistema sanitario, por mencionar alguno de ellos. Se trata, por tanto, de adaptar la sociedad a los cambios demográficos acontecidos sin por ello rasgarse las vestiduras. Dos de los problemas sociales a los que tendrá que enfrentarse una población envejecida serán, por un lado, garantizar en el futuro un sistema de pensiones o de protección social y, por otro, la financiación de la atención sanitaria. El gasto público social ha experimentado un fuerte incremento en los capítulos destinados a los grupos de población de más edad, lo que ha obligado a los Estados del Bienestar a plantearse, por un lado, la necesidad de aumentar sus presupuestos para hacer frente a las necesidades y exigencias de la población y, por otro, a reestructurar la distribución de su gasto público de acuerdo con la cambiante estructura de la misma. Por ejemplo, en la mayoría de los países de la OCDE, el gasto destinado a las personas mayores de 65 años es más de dos veces superior al destinado a los menores de 16 años.

Con el alargamiento de la vida, la calidad de la misma y las condiciones de salud de las personas cobran un especial interés, al igual que un incremento importantísimo de los recursos y los costes sanitarios. Los problemas de salud se traducen en un aumento del gasto público, y con el aumento de las personas mayores de 65 años se incrementa la demanda de servicios sanitarios. La salud constituye un problema esencial, sobre todo en las personas de más edad. No debemos olvidarnos de esa máxima de la Organización Mundial de la Salud (OMS) de que no sólo hay que añadir años a la vida, lo cual es muy importante, sino también, y sobre todo, hay que tratar de añadir vida a los años; es decir, calidad de vida.

Las cuestiones demográficas guardan una estrecha relación con los problemas de la salud y de la enfermedad, y la salud es un problema de primera magnitud. Efectivamente, asociar vejez con enfermedad forma parte del pasado y hay que tratar de desmitificar la tendencia a relacionar y a asociar el envejecimiento con la vejez. No toda persona que llegue a viejo necesariamente tiene que contraer alguna enfermedad. De hecho, cada vez es más frecuente encontrar a personas de 70 y 80 años en perfectas condiciones de salud. La nueva imagen de la vejez tiene poco o nada que ver con la imagen tradicional y estereotipada de personas enfermas, tristes, solitarias, dependientes y sin recursos.

Por otro lado, hay que tener en cuenta que cuando se habla de vejez o de viejos en términos generales estamos refiriéndonos a una situación social o a un sector de la población cuyo único denominador común es pertenecer a una determinada edad socialmente definida como vejez. Muchas son, sin embargo, las diferencias que podemos encontrar: diversos estados de salud física y mental; diferentes grupos de edad y, por lo tanto, diferencias en sus procesos de socialización; distintos niveles educativos; diferentes situaciones de poder adquisitivo y estilos de vida diversos.

Sin embargo, y pese a que ni la enfermedad, ni la soledad, ni la dependencia sean consecuencia del envejecimiento, es en los grupos de la población en edades más avanzadas donde en mayor medida encontramos situaciones caren- 
ciales. Son muchos los estudios empíricos en los que encontramos la relación entre la variable edad y el riesgo a padecer enfermedades o discapacidades.

Una de las principales preocupaciones de las personas mayores es la salud. No en vano a partir de edades avanzadas los enfermos no cesan de crecer, y son los de más edad quienes, con más frecuencia que el resto de la población, sufren problemas de salud. El riesgo de padecer una enfermedad, una incapacidad o una deficiencia aumenta con la edad, lo cual no significa que la edad sea la única variable determinante, pero sí es una variable fundamental. Las variables socioeconómicas tales como el nivel educativo, la profesión y los ingresos familiares son, junto con la edad, variables igualmente importantes a la hora de analizar el riesgo a padecer discapacidades o enfermedades.

Muchas enfermedades no son consecuencia o propias de la vejez, pero presentan en ella aspectos particulares que dan lugar a que sean descritas como patologías de la vejez, bien por su frecuencia, su prolongación o por su supervivencia en estas edades. Un alto porcentaje de personas viejas, aunque no estén enfermas, sufren, sin embargo, algún tipo de discapacidad. Según las Encuestas de morbilidad hospitalaria, los enfermos mayores de 65 años permanecen en los hospitales mucho más tiempo que el resto de la población por la misma enfermedad.

En resumen, los cambios en la estructura de la población están provocando un cambio en la estructura de la morbilidad. Estas son algunas de las preguntas que nos podríamos plantear, a modo de conclusión: la tendencia hacia una disminución de la mortalidad a edades avanzadas, ¿tendrá efectos beneficiosos o perjudiciales para la salud de la población?; ¿puede actualmente el sistema sanitario de los países desarrollados, o podrá en el futuro, hacer frente a las necesidades de salud de las personas en edades avanzadas?

Dos son al menos las posibles respuestas que a modo de hipótesis se barajan entre los estudiosos del tema. La primera hipótesis, y hasta ahora la más compartida, es la de la expansión de la morbilidad. A medida que una población más amplia sobreviva hasta edades avanzadas, aumentarán las posibilidades de enfermedades senescentes conocidas e incluso surgirán otras nuevas asociadas con la edad. Consecuentemente, la demanda de servicios sanitarios seguirá en aumento.

La segunda hipótesis, más novedosa y esperanzadora pero todavía cuestionada, es la de la compresión de la morbilidad (J. Fries, 1980, 1987). Mediante la adopción de mejores estilos de vida y los avances en la tecnología médica, tanto las enfermedades mortales como las degenerativas de la edad avanzada pospondrán su edad de inicio a un período de tiempo más corto, próximo al límite biológico de la especie humana. Según esta hipótesis, al retrasarse la aparición de enfermedades crónicas y la incapacidad, el gasto público en salud no se verá incrementado.

Ambas hipótesis son revisadas por Olshansky y sus colegas (1991, 1993). Para los autores, se ha producido, en los países desarrollados y a lo largo del presente siglo, un descenso en las enfermedades asociadas a edades jóvenes, 
infecciosas o parasitarias, y un progresivo aumento de las enfermedades crónicas de la vejez, unas de consecuencias fatales, como las cardiopatías, los accidentes vasculares o el cáncer, y otras no mortales pero incapacitadoras, como la artritis, las demencias o la osteoporosis. A una de estas enfermedades, no mortales pero sí seriamente inhabilitadoras, dedicaremos los próximos apartados.

\section{LA DEMENCIA TIPO ALZHEIMER. UNA INVESTIGACION SOCIOLOGICA}

Hasta no hace muchos años, la enfermedad de Alzheimer ha sido una gran desconocida, no sólo para la sociedad en general, sino incluso para algunos profesionales de la Medicina. Sin embargo, el interés por la enfermedad crece, a medida que el número de afectados también lo hace.

Desde que, en 1907, Alois Alzheimer describiera las características de la enfermedad hasta la actualidad, muchos han sido los hallazgos científicos para la comprensión de este mal. Pero, quizá, fue el Congreso celebrado en Londres en 1977 el acontecimiento decisivo para que la sociedad en general y la comunidad científica en particular tomaran conciencia de la importancia presente y futura de esta enfermedad.

Una buena prueba de este creciente interés por parte de los científicos quedaba patente en un reciente artículo, publicado por uno de nuestros diarios nacionales, en el que, bajo el título "Los magníficos del 92», se publicaba una selección de los siete campos de investigación y publicaciones científicas de mayor impacto en el año 1992, realizada por la Base de Datos del Institute for Scientific Information. El estudio de proteinas amiloides en la enfermedad de Alzheimer ocupaba un lugar entre esas siete áreas de mayor interés científico, medido por el número de citas en publicaciones científicas de alcance internacional.

La enfermedad de Alzheimer se ha convertido en «una de las principales amenazas sanitarias del siglo XX», en palabras de los expertos que en el mes de marzo de 1993 se reunieron en Madrid para debatir sobre las causas y las consecuencias de este mal. Para el profesor Cacabelos, director de esas Jornadas Internacionales, la enfermedad de Alzheimer se está convirtiendo en «el tercer problema de salud en los países desarrollados».

A juicio de los especialistas, el problema de la enfermedad de Alzheimer es fundamentalmente sanitario y social. Dada la falta de recursos e infraestructuras, son muy pocos los enfermos que están institucionalizados, por lo que la mayor parte de su coste corre a cargo de los propios enfermos o de sus familias.

La enfermedad de Alzheimer es la demencia más frecuente. Se cifra en torno al 60 por 100 del total de las mismas. Su etiología es desconocida, aunque la influencia familiar aparece en más de la mitad de los casos. Su mecanismo fisiopatológico parece consistir en una destrucción neuronal como consecuencia de la lisis de su membrana celular. Esta lisis se produciría debido a la 
síntesis de la Acetil Colina que le falta a la célula a partir de los elementos de la membrana celular. Aunque el sistema que se afecta por este mecanismo es, sobre todo, el colinérgico, también aparecen alterados los sistemas noradrenérgico, dopaminérgico, etc. Esta alteración global de la comunicación sináptica a distintos niveles del sistema nervioso diferencia a la enfermedad de Alzheimer de otras demencias de alteración neurológica como el Parkinson.

La afectación clínica fundamental de esta demencia se localiza sobre todo a nivel de las capacidades cognitivas. Comienza por una alteración de memoria, que progresa en el tiempo y, al final, se generaliza a las restantes capacidades mentales. El enfermo termina con una dependencia total del entorno, y muere frecuentemente como consecuencia de los trastornos ocasionados. El coste social y familiar de esta enfermedad se ve agravado por la relativa larga evolución de la misma.

Ante un problema de tal envergadura, parece imprescindible detectar no sólo el número actual de enfermos, sino también la evolución más probable dentro de esa tendencia progresivamente creciente de la enfermedad dada la actual dinámica demográfica de la población. En este sentido, pretendemos con la presente ponencia profundizar en el análisis de los aspectos epidemiológicos básicos de la enfermedad de Alzheimer en la provincia de Alicante.

Para medir la frecuencia de una enfermedad se suelen utilizar dos conceptos: la incidencia y la prevalencia. La incidencia consiste en el número de personas que contraen una enfermedad en una población determinada, durante un período de tiempo, normalmente un año. La tasa de incidencia mide, por lo tanto, el número de nuevos casos de una enfermedad que se desarrollan en esa población y en ese año en relación con el total de la población o con la población de riesgo. La prevalencia hace referencia al número de personas que padecen una determinada enfermedad en una población concreta, en un corte de tiempo. La tasa de prevalencia es el índice que mide el número de casos de una enfermedad que existe en una población en un momento dado respecto a la población total o población de riesgo.

La incidencia es un indicador más sensible que la prevalencia y nos indica la probabilidad que tiene una población de contraer una determinada enfermedad. Los estudios de la incidencia de las enfermedades nos proporcionan una medida muy exacta de las necesidades médicas y sanitarias de un país. También es un indicador más útil que la prevalencia en el caso de enfermedades crónicas cuya etiología todavía está por aclarar, como es el caso de la enfermedad de Alzheimer. Sin embargo, en algunas enfermedades, como la que aquí nos ocupa, al no ser de declaración obligatoria, no existen registros oficiales y, por tanto, la incidencia, como la prevalencia, resultan muy difíciles de calcular con exactitud. Es por esa razón por la que no existen una gran cantidad de estudios sobre la frecuencia de la enfermedad de Alzheimer.

Los estudios epidemiológicos de las demencias en general presentan diversos problemas metodológicos derivados de la fiabilidad del diagnóstico, de los criterios utilizados para definir el grado de deterioro o de la distinción entre 
los enfermos institucionalizados y los que viven en sus domicilios, entre otros. Los distintos procedimientos utilizados para la recogida de datos y los distintos criterios diagnósticos empleados hacen que los estudios epidemiológicos realizados en diversos países lleguen a índices de prevalencia muy variados. La OMS señala que los índices publicados sobre las demencias suaves y moderadas, por estas razones anteriormente expuestas, oscilan entre el 3 y el 30 por 100 para las personas mayores de 65 años. Para las demencias en estados más avanzados, los índices oscilan entre el 5 y el 8 por 100. El riesgo de presentar una demencia grave a los 80 años se estima entre el 15 y el 20 por 100 (Barcelona, 1992).

Sin embargo, en los estudios epidemiológicos sobre demencias que han utilizado parecidos procedimientos de recogida de datos y de criterios diagnósticos las oscilaciones son menores: los datos de prevalencia oscilan entre el 5 por 100 (hipótesis baja), el 6,3 por 100 (hipótesis media) y el 7,1 por 100 (hipótesis alta) para las personas de 65 y más años. La progresión de la prevalencia a los 80-85 años es considerable y afecta, sobre todo, a las mujeres (Hugonot, 1988). Estos índices han sido utilizados en Barcelona por el laboratorio de Sociología del ICESB (1992) para estimar la demencia senil y son los que vamos a utilizar nosotros para la estimación de la prevalencia de las demencias en la provincia de Alicante.

Los estudios epidemiológicos de las demencias en España empiezan a ser frecuentes, sobre todo en la última década. Bermejo y Tolosa (1990) recogen información de diversos estudios, acabados unos y en marcha otros, en las ciudades de Zaragoza, Pamplona, Palma de Mallorca, Barcelona, Gerona y Madrid. Sin embargo, los estudios concretos sobre epidemiología de la enfermedad de Alzheimer son escasos. Según estos estudios, la tasa de prevalencia de demencia variaría desde un 5,2 por 100 en el estudio de Zaragoza (Lobo, 1989) hasta un 13,5 por 100 según el estudio de Madrid (Colmenarejo, 1989).

La enfermedad de Alzheimer representa, para algunos, entre el 55 y el 75 por 100 de los casos de demencia. La prevalencia de las demencias de tipo Alzheimer en las personas mayores de 65 años es, probablemente, del 5 por 100 . El aumento exponencial de la prevalencia con la edad es un hecho sobre el que existe una gran unanimidad. La prevalencia se sitúa entre el 0,1 por 100 antes de los 65 años, el 1,1 por 100 entre los de 65 y 74 años y, posiblemente, superior al 10 por 100 en los de más de 85 años.

La incidencia anual de la demencia tipo Alzheimer, aunque difícil de apreciar, dada la no declaración obligatoria de la enfermedad y, por tanto, de los nuevos casos, se sitúa alrededor del 3 por 1.000. En la mayoría de los estudios se destaca una prevalencia mayor de las mujeres, sin que haya sido determinada si es o no real; un aumento exponencial con la edad, como hemos visto anteriormente, y una supervivencia media de 8,1 años, lo que supone una reducción de la esperanza de vida de estos enfermos (Thomas et al., 1990).

Según el doctor Cacabelos, después de analizar diversos estudios epidemio- 
lógicos concluye que, asumiendo una media del 60 por 100 de enfermedad de Alzheimer entre todas las demencias, se estima que la prevalencia de esta enfermedad suele ser del 4,2 por 100 en los mayores de 65 años. Estos índices son los que utilizaremos para calcular la prevalencia de la enfermedad de Alzheimer en la provincia de Alicante.

La enfermedad de Alzheimer afecta a 20 millones de personas en todo el mundo y, según los expertos, de no encontrarse un tratamiento eficaz, la enfermedad podría duplicar este número para el año 2025, dado el progresivo envejecimiento de la población. En España afecta a más de 300.000 personas, mientras que hace un año los especialistas cifraban en 200.000 el número de personas afectadas. En el Reino Unido, esta llamada por algunos «marea creciente» afectaba a 750.000 personas. En Estados Unidos, cerca de un millón y medio de personas la padecen, ocupando el cuarto lugar entre las causas de muerte. En Canadá y en Francia, los enfermos de Alzheimer superan los 300.000 casos.

Respecto a estudios epidemiológicos sobre la demencia tipo Alzheimer, W. Rocca (1993) realizó una actualización y revisión de los datos y teorías más importantes sobre la frecuencia, distribución y factores de riesgo de la enfermedad de Alzheimer, extraídos de una serie de estudios, realizados con distintas metodologías, en distintos países europeos, EE.UU. e Israel. Las conclusiones a las que llega Rocca son las siguientes:

- La incidencia y la prevalencia de la enfermedad de Alzheimer son generalmente mayores en las mujeres que en los hombres. En diversos estudios, estas diferencias se producen en todos los grupos de edad.

- La frecuencia de la enfermedad de Alzheimer aumenta con la edad en todos los países analizados.

- Es escasa la diferencia de la frecuencia de la enfermedad de Alzheimer entre distintos países, incluso entre distintos continentes.

Animados al estudio de esta enfermedad que más frecuentemente se asocia al envejecimiento pues, según todos los expertos, actúa y aparece con una mayor frecuencia entre la población mayor de 65 años, nos dispusimos a la investigación de la incidencia, presente y futura, de la enfermedad de Alzheimer en nuestra provincia.

ESTIMACION DE LA INCIDENCIA DE LA ENFERMEDAD DE ALZHEIMER EN LA PROVINCIA DE ALICANTE

Para estimar la incidencia de la enfermedad de Alzheimer en la provincia de Alicante, consideramos que habría que reproducir el camino que sigue normalmente el enfermo en su evolución a través del sistema sanitario. Por esta 
razón decidimos que la mejor forma de conseguir nuestro objetivo era sondear dos poblaciones de médicos. La primera de éstas vendría constituida por los médicos generales. Estos son los primeros profesionales que entran en contacto con el enfermo y después siguen su evolución. Aunque su contribución al diagnóstico de la enfermedad no es definitiva, sí pueden orientar un diagnóstico de sospecha. La complejidad de esta patología, los tests de mayor o menor dificultad y el nivel de aparataje necesario alejan del médico general la posibilidad de realización de un diagnóstico definitivo. Sin embargo, como de su competencia para detectar la patología de Alzheimer depende la precocidad de las medidas a tomar, su opinión nos pareció fundamental. Además, esta postura nos permitiría analizar la interacción entre el enfermo de Alzheimer y el sistema sanitario en toda su complejidad.

El otro grupo de población quedó definido como médicos especialistas. Dentro del mismo incluimos a todos aquellos que, por razón de su contacto con este tipo de enfermos, se ven abocados a realizar el diagnóstico o contribuyen al mismo de una forma definitiva. Entre ellos consideramos a los psiquiatras, neurólogos y geriatras como principales especialistas en relación con la enfermedad.

Para el estudio y recogida de datos relevantes en el cálculo de la incidencia de la enfermedad, consideramos que la encuesta era la técnica más adecuada. En el desarrollo práctico de la misma se eligió la entrevista telefónica como la técnica que mejor se adaptaba a nuestras metas y condiciones. Esta decisión quedaba avalada por el hecho de que el cuestionario hacía referencia a un número muy limitado de preguntas. Esta es una de las condiciones fundamentales para la realización de las entrevistas de tipo telefónico. Otra de las razones que impulsó esta decisión se encontraba en la falta de recursos suficientes para el uso de una red de campo. La sencillez de la información que se requería también fue un factor de peso en esta decisión. En último lugar, la entrevista telefónica permitía asegurar en un máximo grado la aleatoriedad en la elección de la muestra. El único problema podría consistir en la carencia de teléfono de alguno de los facultativos, pero esta situación fue más que excepcional.

Para llevar a cabo la distribución aleatoria del cuestionario desarrollamos los siguientes pasos. En primer lugar calculamos el tamaño de la muestra. Para un total de 2.720 médicos generales y 108 especialistas (geriatras, neurólogos y psiquiatras), el tamaño de la muestra es de 191 para los médicos generales y de 24 para los médicos especialistas para un intervalo de confianza del 95 por 100 y un error muestral de \pm 2 sigma. Los datos de los médicos seleccionados en cada uno de los universos se obtuvieron a través de un listado de profesionales colegiados cedido gentilmente por el Colegio Oficial de Médicos de la provincia de Alicante.

A continuación agrupamos los municipios de la provincia, según su tamaño de población, en nueve grupos y asignamos un número de médicos proporcional al tamaño de cada uno. Posteriormente diseñamos un cuestionario inte- 
grado por nueve preguntas, en su mayor parte abiertas, que fueron categorizadas a continuación. El cuestionario iba dirigido a médicos generales y a médicos especialistas, con la finalidad de la posterior comparación de resultados.

A través de las dos primeras preguntas del cuestionario intentamos medir la incidencia y la prevalencia de la enfermedad. En primer lugar se les preguntaba si habían diagnosticado algún enfermo de Alzheimer. Si la respuesta era negativa, se acababa la entrevista. Si era afirmativa, se les preguntaba por el número de enfermos diagnosticados dentro del último año. A continuación se les pedía información sobre las edades y el sexo de los diagnosticados, la fase de evolución en que los diagnosticaron, así como las manifestaciones clínicas fundamentales. Finalmente, se les preguntaba si sabían cuántos de estos enfermos habían sido internados y cuántos permanecían en sus domicilios.

La información de los médicos generales, esto es, la más sensible, nos puede detectar todos los enfermos de Alzheimer que existen en la provincia de Alicante. Sin embargo, esto puede suponer el diagnóstico como demencias tipo Alzheimer de algunas demencias que no lo son, como, por ejemplo, demencias arteriopáticas, demencias seniles, etc., esto es, nos van a aumentar el número de falsos positivos.

Por el contrario, la estimación realizada por los médicos especialistas es la más fiable. Esto quiere decir que los casos que se hayan detectado como enfermos de Alzheimer lo son en realidad. Sin embargo, el problema que tiene esta estimación es que vamos a desechar algunos casos que, siendo enfermos de Alzheimer, no los hemos considerado como tales. Esto es, nos habrán aumentado los falsos negativos.

La realidad, por tanto, estaría situada dinámicamente en la frontera entre la estimación de los médicos generales y la estimación de los médicos especialistas. Esta última puede reflejar el mínimo real del número de enfermos, mientras que la primera supone el máximo número de enfermos que podemos detectar en la provincia.

Esta situación se puede explicar por el hecho de que un cierto número de enfermos llegue tardíamente al médico especialista. Por ejemplo, es más fácil que en el medio rural, y en el caso de personas sin una actividad intelectual, la sintomatología de la enfermedad pueda pasar desapercibida, al ser catalogada como demencia senil o problemas propios de la vejez. Se ocasionaría, por ello, la llegada tardía de estos enfermos al médico especialista. Habría, por tanto, durante un cierto tiempo, un relativo número de enfermos que serían detectados por el médico general y no por el especialista. Esto puede explicar que, durante el tiempo en que se hizo la estimación, hubiera un número mayor de enfermos diagnosticados por los médicos generales que por los especialistas.

A continuación presentamos nuestras conclusiones respecto a la estimación de la incidencia de la enfermedad de Alzheimer en la provincia de Alicante.

— Total de médicos generales de la provincia de Alicante: 2.711.

- Total de médicos especialistas de la provincia de Alicante: 108. 
- Entrevistas realizadas a médicos generales: 191.

- Entrevistas realizadas a médicos especialistas: 24.

— Total de entrevistas telefónicas realizadas: 215.

- Entrevistas válidas a médicos generales: 80 .

- Entrevistas válidas a médicos especialistas: 16.

— Total de entrevistas válidas: 96.

- Número de médicos generales que han diagnosticado enfermos de Alzheimer en nuestra muestra: de los 80 médicos, 24 médicos no han diagnosticado y 7 no saben/no contestan; por tanto, 49 médicos han diagnosticado algún enfermo de A. (véase tabla 1).

- Las razones por las cuales no han diagnosticado ningún enfermo son, en la mayoría de los casos, por el tipo de actividad que estaban realizando en el momento de la entrevista: trabajo en urgencias, tener una especialidad ajena a esta enfermedad y no figurar como tal en el listado del Colegio de Médicos, estar jubilados, estar en paro o, simplemente, no haber diagnosticado a ninguno.

- Número de médicos especialistas que han diagnosticado enfermos de Alzheimer en nuestra muestra: de los 16 especialistas, 2 no han diagnosticado y ninguno no sabe/no contesta; por tanto, 14 especialistas han diagnosticado algún enfermo de Alzheimer (véase tabla 2).

- Porcentaje de médicos generales de nuestra muestra que han diagnosticado enfermos de Alzheimer: 61 por 100.

- Porcentaje de médicos especialistas de nuestra muestra que han diagnosticado enfermos de Alzheimer: 87 por 100.

- Si el 61 por 100 de médicos generales de nuestra muestra han diagnosticado enfermos de Alzheimer, se puede considerar que al haber sido seleccionada por muestreo aleatorio simple, también el 61 por 100 del total de médicos generales de la provincia de Alicante debería haberlos diagnosticado.

- Si el 87 por 100 de médicos especialistas de nuestra muestra han diagnosticado enfermos de Alzheimer, se puede considerar que al haber sido seleccionada por muestreo aleatorio simple, también el 87 por 100 del total de especialistas de la provincia de Alicante debería haberlos diagnosticado.

- Si aplicamos el porcentaje de médicos generales de la muestra (61 por 100) al total de médicos generales de la provincia de Alicante, resulta que 1.653 han diagnosticado algún enfermo de Alzheimer.

- Si aplicamos el porcentaje de médicos especialistas de la muestra (87 por 100) al total de médicos especialistas de la provincia de Alicante, resulta que 93 han diagnosticado algún enfermo de Alzheimer. 


\section{TABLA 1}

Incidencia de la EA para los médicos generales

\begin{tabular}{|c|c|}
\hline $\begin{array}{c}\text { Enfermos diagnosticados } \\
\text { en el último año }\end{array}$ & Médico general \\
\hline ......... & 24 \\
\hline . & 12 \\
\hline 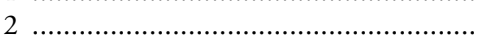 & 17 \\
\hline 3 & 8 \\
\hline (n) & 4 \\
\hline 5 & 3 \\
\hline 9 . & 1 \\
\hline 20 & 3 \\
\hline 30 & 1 \\
\hline 99 & 7 \\
\hline${ }_{1}$ & 80 \\
\hline
\end{tabular}

FUENTE: Elaboración propia, Alzheimer, 93.

\section{TABLA 2}

Incidencia de la EA para los médicos especialistas

\begin{tabular}{|c|c|}
\hline $\begin{array}{l}\text { Enfermos diagnosticados } \\
\text { en el último año }\end{array}$ & Médico especialista \\
\hline $0 \ldots \ldots \ldots \ldots \ldots$ & 2 \\
\hline 2 & 1 \\
\hline З & 5 \\
\hline 4 … & 1 \\
\hline 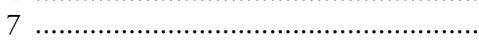 & 1 \\
\hline 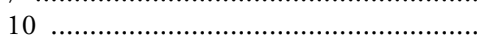 & 1 \\
\hline 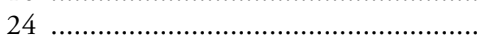 & 1 \\
\hline 30 & 1 \\
\hline 35 & 1 \\
\hline 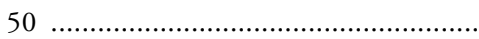 & 1 \\
\hline 60 & 1 \\
\hline 99 & 0 \\
\hline Total & 16 \\
\hline
\end{tabular}

FUENTE: Elaboración propia, Alzheimer, 93. 
Para calcular el número de enfermos que ha sido diagnosticado por cada grupo de médicos hemos considerado la posibilidad de varios estadísticos. Entre ellos elegimos como el más apropiado la mediana, porque es la que menos se ve influida por los valores extremos de nuestra distribución. A pesar de ello, hemos desestimado los valores 0 y 30 en la tabla 1 y 0,50 y 60 en la tabla 2, porque son valores excesivos y poco verosímiles. Los valores 99, correspondientes a no sabe/no contesta, se filtran, puesto que nuestro objetivo es detectar los médicos que han tenido contacto con enfermos de Alzheimer. Como puede verse en las tablas 3 y 4 , la mediana de la frecuencia de las categorías referentes al número de enfermos vistos por los médicos generales es 2 . La mediana de los médicos especialistas es 5,5.

\section{TABLA 3}

Estadísticos para la estimación de la incidencia en médicos generales

\begin{tabular}{|c|c|}
\hline \multicolumn{2}{|c|}{ Enfermos diagnosticados de EA en el último año } \\
\hline 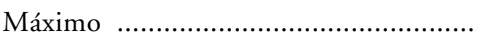 & 20 \\
\hline 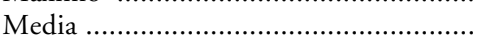 & 4 \\
\hline 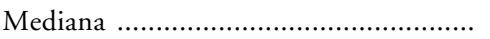 & 2 \\
\hline 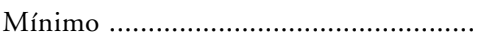 & 1 \\
\hline 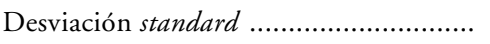 & 5 \\
\hline
\end{tabular}

FUENTE: Elaboración propia, Alzheimer, 93.

\section{TABLA 4}

\section{Estadisticos para la estimación de la incidencia en médicos especialistas}

\begin{tabular}{|c|c|}
\hline \multicolumn{2}{|c|}{ Enfermos diagnosticados de EA en el último año } \\
\hline Máximo & 35 \\
\hline Media & 17 \\
\hline 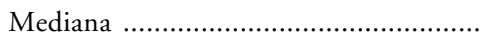 & 5 \\
\hline Mínimo & 2 \\
\hline 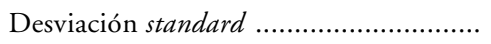 & 4 \\
\hline
\end{tabular}

FUENTE: Elaboración propia, Alzheimer, 93. 
Por consiguiente, podemos considerar que los 1.653 médicos generales han diagnosticado un total de $1.653 \times 2=3.306$ enfermos en los últimos doce meses.

De la misma forma, podemos considerar que los 93 médicos especialistas han diagnosticado un total de $93 \times 5,5=511$ enfermos en los últimos doce meses.

Como vemos, hay dos estimaciones que varían en sus resultados. Una de ellas, la de los médicos especialistas, es más fina. Se aproxima mucho a la tasa de incidencia del 3 por 1.000, sobre la que existe bastante acuerdo entre los diversos estudios epidemiológicos realizados. Si consideramos que la población de riesgo en la provincia de Alicante es de 166.473 personas, resulta que la incidencia con respecto a este 3 por 1.000 supone 499 . Cifra muy próxima a los 511 enfermos de Alzheimer que se reflejan en nuestra estimación de médicos especialistas.

La estimación realizada por los médicos generales, por el contrario, ofrece una cifra de enfermos mucho mayor. Esto se podría explicar como consecuencia de que éstos realizan diagnósticos de menor fiabilidad que los especialistas. Además, podría pensarse que los médicos generales, al tener un concepto más difuso de la enfermedad, dado el mayor abanico de enfermedades que tratan, recordasen un número de casos mayor que los reales. Otra causa que explique este hecho puede ser que algunos médicos declararan algunos casos como de primer diagnóstico cuando en realidad se trataría de pacientes anteriormente diagnosticados.

\section{Edad de diagnóstico}

Una de las preguntas del cuestionario pretendía obtener información de los médicos generales y de los especialistas acerca de la edad en la que suelen diagnosticar a sus pacientes de Alzheimer. Dado que muchos médicos no tienen un registro exhaustivo de sus enfermos, recordar la edad en la que fueron diagnosticados les resultaba prácticamente imposible. Hay que señalar que en esta pregunta los médicos no elegían una única opción, sino que siempre se referían a los dos grupos de edad que diagnosticaban con más frecuencia. De ahí que los porcentajes de la tabla no vayan referidos al 100 por 100 , sino al 50 por 100 , ya que la pregunta admite a la vez dos respuestas. Estos datos aparecen en la tabla 5 .

A través de los datos que presentamos en esta tabla se puede apreciar que una gran mayoría de los médicos (51 por 100$)$, sobre todo generales (46,4 por $100)$, o bien no saben la edad de sus pacientes o bien en ese momento no la recordaban.

Existe una gran unanimidad entre los médicos generales y los especialistas (concretamente un 22,4 por 100 del total de médicos) en que el grupo de edad donde se diagnostica un mayor número de enfermos de Alzheimer es entre los 61 y 70 años.

Tanto los médicos generales como los especialistas diagnostican un reduci- 


\section{TABLA 5}

Edad de diagnóstico (\%)

\begin{tabular}{|c|c|c|c|}
\hline \multirow[b]{2}{*}{ Edad de diagnóstico } & \multicolumn{2}{|c|}{ Médicos } & \multirow[b]{2}{*}{ Total de casos } \\
\hline & Generales & Especialistas & \\
\hline 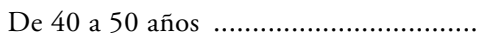 & 2,1 & 2,6 & 4,7 \\
\hline 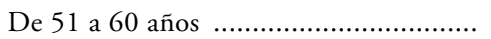 & 8,9 & 3,1 & 12,0 \\
\hline De 61 a 70 años & 17,5 & 5,2 & 22,7 \\
\hline Más de 70 años & 8,5 & 1,0 & 9,5 \\
\hline 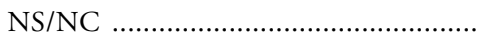 & 46,4 & 4,7 & 51,0 \\
\hline 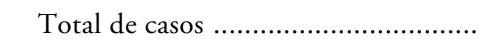 & 41,7 & 8,3 & 50,0 \\
\hline
\end{tabular}

FUENTE: Elaboración propia, Alzheimer, 93.

do número de enfermos entre las categorías extremas de nuestra tabla. Esta situación es normal, dado que la edad de presentación más frecuente de la enfermedad de Alzheimer es a partir de los 60 años.

\section{Predominio de sexo en la enfermedad de Alzheimer}

En la mayor parte de la literatura se describe el predominio del sexo femenino sobre el masculino en la enfermedad de Alzheimer. Este dato se refleja claramente en la tabla 6.

\section{TABLA 6}

Predominio del sexo en la EA

\begin{tabular}{|c|c|c|c|}
\hline & \multicolumn{2}{|c|}{ Médicos } & \multirow[b]{2}{*}{ Total } \\
\hline & Generales & Especialistas & \\
\hline Más mujeres que hombres .................... & 44,6 & 56,2 & 47,2 \\
\hline 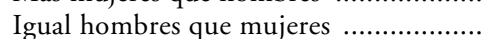 & 12,5 & 37,5 & 33,3 \\
\hline Más hombres que mujeres .................... & 41,1 & 6,3 & 18,1 \\
\hline 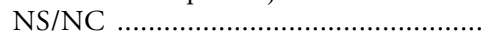 & 1,6 & - & 1,3 \\
\hline
\end{tabular}

FUENTE: Elaboración propia, Alzheimer, 93. 
Conviene indicar que los porcentajes presentados no vienen referidos a enfermos, sino a médicos. Así, por ejemplo, el 47,2 por 100 de los médicos encuentra un predominio del sexo femenino, pero sin cuantificar en qué medida se da este predominio. Esta situación responde al hecho de que los médicos no tenían esta información en sus datos sobre la enfermedad. Mientras que en los médicos generales la opinión sobre el predominio de sexo en la enfermedad de Alzheimer se distribuye casi en partes iguales, en los médicos especialistas se muestra un claro predominio de los que consideran más frecuente el sexo femenino en la enfermedad.

\section{Fase de diagnóstico de la enfermedad}

Tanto la especialidad del médico como la residencia en el medio rural o urbano influyen en la fase en que el enfermo de Alzheimer es diagnosticado. Según nuestros resultados, se puede observar que mientras algo más del 30 por 100 de los médicos generales ve a los enfermos de Alzheimer en fases iniciales de la enfermedad, más del 50 por 100 los ve en fases avanzadas. Por el contrario, los médicos especialistas muestran una pauta diagnóstica más equilibrada en las distintas fases evolutivas de la enfermedad. A pesar de ello, se manifiesta de forma clara que se diagnostican menos enfermos en fases avanzadas de la enfermedad.

Con respecto a la influencia del tamaño de la población en la fase evolutiva en que se diagnostica al enfermo, podemos constatar una serie de hechos. La fase evolutiva en que se diagnostica la enfermedad va a estar influida por la diferencia rural-urbano. En este último medio, la mejor accesibilidad al médico especialista favorece que el enfermo sea visto por éste en fases iniciales. El médico general, al encargarse principalmente de la terapia de mantenimiento, va a entrar en contacto con el enfermo sobre todo en fases más avanzadas. Mientras que casi el 70 por 100 de los enfermos procedentes de poblaciones rurales son diagnosticados en fase avanzada de la enfermedad, en torno a un 20 por 100 lo son en fase inicial. Por el contrario, sus compañeros en medios urbanos como Elche y Alicante diagnostican en torno a la mitad de los casos de la enfermedad en fase inicial (casi un 50 por 100) y sólo algo menos del 10 por 100 en fase avanzada.

En nuestra muestra hay un predominio relativo de médicos generales en el medio rural con respecto al urbano. Esto es, si consideramos la relación fase inicial/fase avanzada, en la pauta de diagnóstico rural de los médicos generales esta relación es 20 por $100 / 70$ por 100 , mientras que en la pauta urbana es aproximadamente 50 por $100 / 10$ por 100 . 


\section{Manifestaciones clinicas}

Las manifestaciones clínicas fundamentales de los enfermos de Alzheimer son detectadas con pautas de frecuencia similares tanto por los médicos generales como por los especialistas. Como se puede ver en la tabla 7, coinciden las manifestaciones clínicas más frecuentes en ambos grupos. Así, la amnesia y la agnosia son las dos manifestaciones clínicas más frecuentes en ambos tipos de médicos. Aunque sus porcentajes de frecuencia no coincidan, sí lo hacen las manifestaciones más frecuentes. Esto se puede explicar por el hecho de que ésta es una pregunta que puede admitir cuatro respuestas simultáneas. También se explica por esta razón el hecho de que en la suma total de casos aparezca el 25 por 100 (el porcentaje correspondiente a cada una de las respuestas).

\section{TABLA 7}

Manifestaciones clinicas (\%)

\begin{tabular}{|c|c|c|c|}
\hline \multirow[b]{2}{*}{ Manifestaciones clinicas } & \multicolumn{2}{|c|}{ Médicos } & \multirow[b]{2}{*}{ Total de casos } \\
\hline & Generales & Especialistas & \\
\hline Amnesia ... & 9,4 & 2,3 & 11,7 \\
\hline 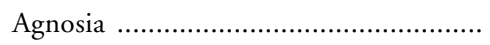 & 8,3 & 2,3 & 10,7 \\
\hline Apraxia & 3,1 & 1,3 & 4,4 \\
\hline Afasia & 3,6 & 1,0 & 4,7 \\
\hline Alteración comportamiento .................... & 4,9 & 1,3 & 6,3 \\
\hline Alteración conciencia ............................... & 2,1 & 0,5 & 2,6 \\
\hline 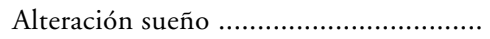 & 0,8 & 0,3 & 1,0 \\
\hline Alteración neuromotoras .......................... & 2,1 & 0,3 & 2,3 \\
\hline 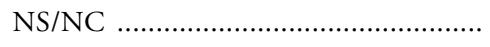 & 49,0 & 7,3 & 56,3 \\
\hline 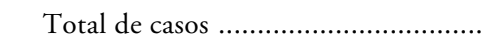 & 20,8 & 4,2 & 25,0 \\
\hline
\end{tabular}

FUENTE: Elaboración propia, Alzheimer, 93.

\section{ESTIMACION DE LA PREVALENCIA DE LAS DEMENCIAS Y DE LA ENFERMEDAD DE ALZHEIMER EN LA PROVINCIA DE ALICANTE}

Ya se ha hablado del incremento del número absoluto y relativo de las personas de más de 65 años en los países occidentales. La provincia de Alicante no es ajena a este fenómeno y su tasa de envejecimiento es muy similar a la media española y europea, aunque ligeramente inferior. En la provincia de Alicante, según los datos del último Censo de Población de 1991, el porcentaje de per- 
sonas mayores de 65 años respecto del total de la población era del 12,8 por 100. Es interesante destacar el desequilibrio existente entre los dos sexos: mientras que la tasa de envejecimiento masculina en la provincia de Alicante es del 11,1 por 100 , la femenina es del 14,5 por 100 . Como puede apreciarse en la tabla 8 , la población femenina es superior a la masculina en todos los grupos de edad.

\section{TABLA 8}

Estructura de la población de 65 años y más de la provincia de Alicante por edad y sexo

\begin{tabular}{|c|c|c|c|}
\hline Edad & Total & Varones & Mujeres \\
\hline $65-69$. & 61.665 & 28.731 & 32.934 \\
\hline 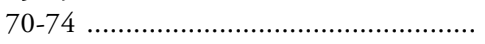 & 42.680 & 18.612 & 24.068 \\
\hline 75-79 & 30.531 & 12.497 & 18.034 \\
\hline 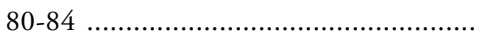 & 19.390 & 7.146 & 12.244 \\
\hline 85 y más & 12.207 & 3.732 & 8.475 \\
\hline 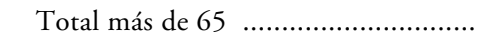 & 166.473 & 70.718 & 95.755 \\
\hline
\end{tabular}

Fuente: INE, Censo de la Población de España, 1991.

Tomando como punto de partida los porcentajes aceptados de prevalencia y adaptándolos a la población mayor de 65 años de Alicante, la población afectada de demencia, según estos datos, sin distinción de sexo y edad, sería:

- Hipótesis baja: 5,0 por 100 (8.323 afectados).

- Hipótesis media: 6,3 por 100 (10.487 afectados).

- Hipótesis alta: 7,1 por 100 (11.819 afectados).

Por lo tanto, el número de habitantes de más de 65 años afectados por demencias en general en la provincia de Alicante se encontraba entre $9.000 \mathrm{y}$ 12.000 personas.

$\mathrm{Si}$ asumimos, siguiendo a Cacabelos, que una media del 60 por 100 de todas las demencias son demencias tipo Alzheimer y adoptamos esa proporción a los datos de las demencias en general, tendríamos una población de enfermos de Alzheimer en la provincia de Alicante de:

- 4.993 según la hipótesis baja.

- 6.292 según la hipótesis media.

- 7.091 según la hipótesis alta. 
Si adoptamos el criterio de que la estimación de la prevalencia de la enfermedad de Alzheimer es del 4,2 por 100 en los mayores de 65 años (Cacabelos, 1991: 21), resultaría una cifra más cercana a la hipótesis alta. Tendríamos una prevalencia de 6.991 enfermos de Alzheimer.

Respecto al futuro de la prevalencia de la enfermedad de Alzheimer en la provincia de Alicante, vamos a utilizar las Proyecciones de la Población masculina y femenina de 65 años y más de la provincia de Alicante, que hemos elaborado a partir de las tasas de mortalidad y de supervivencia, por edad y sexo, de 1991. En las tablas 9, 10 y 11 presentamos estas Proyecciones, que han sido calculadas para los años 1996, 2001 y 2006.

\section{TABLA 9}

Proyección de la población masculina de Alicante por grupos de edad

\begin{tabular}{|c|c|c|c|}
\hline Edad & 1996 & 2001 & 2006 \\
\hline $65-69 \ldots \ldots \ldots$ & 29.613 & 28.002 & 26.125 \\
\hline 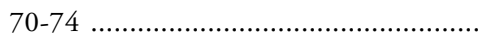 & 24.741 & 25.500 & 24.113 \\
\hline 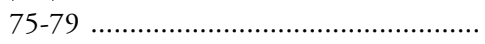 & 14.599 & 19.406 & 20.002 \\
\hline 80-84 & 8.198 & 9.577 & 12.731 \\
\hline 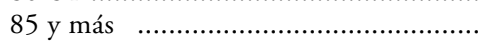 & 4.178 & 4.754 & 5.505 \\
\hline Total & 81.329 & 87.239 & 88.474 \\
\hline
\end{tabular}

Fuente: Elaboración propia a partir de los datos del Censo de Población y de los Movimientos Naturales de Población de 1991.

TABLA 10

Proyección de la población femenina de Alicante por grupos de edad

\begin{tabular}{|c|c|c|c|}
\hline Edad & 1996 & 2001 & 2006 \\
\hline 65-69 ................. & 34.241 & 33.088 & 30.794 \\
\hline 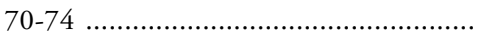 & 30.432 & 31.640 & 30.575 \\
\hline 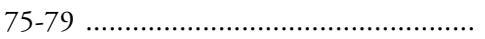 & 20.819 & 26.324 & 27.368 \\
\hline 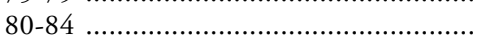 & 13.459 & 15.537 & 19.645 \\
\hline 85 y más & 8.736 & 9.358 & 10.497 \\
\hline Total & 107.686 & 115.947 & 118.879 \\
\hline
\end{tabular}

Fuente: Elaboración propia a partir de los datos del Censo de Población y de los Movimientos Naturales de la Población de 1991. 
En cuanto a la Proyección de la Población de 65 años y más, podemos apreciar su incremento constante y las diferencias en cuanto a sexos. Para el año 2006 se prevé que la población anciana mantenga su crecimiento, sobre todo en los grupos de mayor edad. Es previsible el incremento de la población mayor de 65 años en el primer cuarto del siglo XXI.

Según estos datos, la población de más de 65 años, de mantenerse las tasas de mortalidad y supervivencia del año 1991, será la que presentamos en la tabla 11.

\section{TABLA 11}

Proyección de la población de 65 años y más de Alicante por sexos

\begin{tabular}{|c|c|c|c|c|}
\hline & 1991 & 1996 & 2001 & 2006 \\
\hline 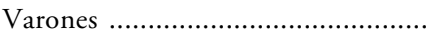 & 70.718 & 81.329 & 87.239 & 88.474 \\
\hline Mujeres & 95.755 & 107.686 & 115.947 & 118.879 \\
\hline (n) & 166.473 & 189.015 & 203.186 & 207.353 \\
\hline
\end{tabular}

Fuente: Elaboración propia a partir de los datos del Censo de Población y de los Movimientos Naturales de la Población de 1991.

Si tenemos en cuenta la proyección de la población total de 65 y más años de la provincia de Alicante desde 1991 a 2006, según las Hipótesis Baja, Media y Alta, la estimación de la posible prevalencia de la demencia en general en la provincia de Alicante será la que aparece en la tabla 12 .

TABLA 12

Proyección de la prevalencia de la demencia en general en Alicante

\begin{tabular}{|c|c|c|c|c|}
\hline & 1991 & 1996 & 2001 & 2006 \\
\hline Hipótesis baja & 8.323 & 9.450 & 10.159 & 10.367 \\
\hline Hipótesis media & 10.487 & 11.907 & 12.800 & 13.063 \\
\hline 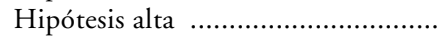 & 11.819 & 13.420 & 14.426 & 14.722 \\
\hline
\end{tabular}

Fuente: Elaboración propia a partir de los datos del Censo de Población de 1991.

Si aplicamos el 60 por 100 (cifra media aceptada por la mayoría de los estudiosos para la enfermedad de Alzheimer) a estos datos de las demencias en general, la proyección de la posible prevalencia de la enfermedad de Alzheimer para la provincia de Alicante desde 1991 al 2006 se ve en la tabla 13. 
TABLA 13

Proyección de la prevalencia de la enfermedad de Alzheimer en Alicante

\begin{tabular}{|c|c|c|c|c|}
\hline & 1991 & 1996 & 2001 & 2006 \\
\hline 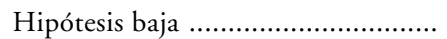 & 4.993 & 5.670 & 6.095 & 6.220 \\
\hline 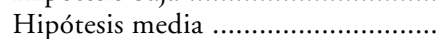 & 6.292 & 7.144 & 7.680 & 7.837 \\
\hline Hipótesis alta & 7.091 & 8.052 & 8.655 & 8.833 \\
\hline
\end{tabular}

Fuente: Elaboración propia a partir de los datos del Censo de Población de 1991.

\section{BIBLIOGRAFIA}

AgID, Y., y BRICE, A. (1993): «La agitada genética de la enfermedad de Alzheimer», en Mundo Cientifico, vol. 13, núm. 139, octubre.

Altimir, S. (1991): Malaltía d'Alzheimer: que ens cal saber, Barcelona: Generalitat de Catalunya, Diari Oficial i de Publicació.

Altimir, S., y GonZÁlez, A. M. (1990): Servicio de ayuda a domicilio a personas con enfermedad de Alzheimer, Barcelona: AFA.

Arbelo, A., et al. (1989): Demografía Sanitaria de la Ancianidad, Madrid: Ministerio de Sanidad y Consumo.

Albareda, J. L., y Micas, M. (1986): «Détection précoce des démences: aspect gérontologique», en Symposium sur la démence sénile, París.

BAhr, S. J., y Peterson, E. T. (1989): Aging and the Family, Lexington, MA: Lexington Books.

BARASH D. (1987): El envejecimiento, Barcelona: Biblioteca Científica Salvat.

Barenys, M. P. (coord.) (1993): «Envelliment i Societat», en Papers. Revista de Sociología, núm. 40.

Bermejo, F. (1987): «Epidemiología de la demencia. Revisión del tema y datos de España», en Revista Clínica Española, núm. 181.

Bermejo, F., y Tolosa, E. (1990): «Epidemiología de las enfermedades neurológicas en el anciano", en Epidemiología del envejecimiento en España, Madrid: Ministerio de Sanidad y Consumo, cap. 4.

Bermejo, P., y Del Ser, T. (1993): Demencias. Conceptos actuales, Madrid: Ed. Díaz de Santos.

BLESA, R. (1987): «Diagnóstico precoz y diagnóstico diferencia de la enfermedad de Alzheimer», en Revista Clínica Española, vol. 181.

Boada, M. (coord.) (1990): Les demències a Catalunya. Pla Pilot d'un model d'atenció sòciosanitària per a malalts amb demència d'Alzheimer $i$ d'altres afins, Barcelona: Generalitat de Catalunya (inédito).

Caballero, J. C. (1987): «Demencia senil: un reto asistencial, sanitario y social», en Sandoz S.A.E., Barcelona.

Caballero, J. C., y SAntamaría, J. (1991): «Demencia y servicios sociales», en Revista Española de Geriatría y Gerontología, núm. 262.

Cacabelos, R. (1989): «Demencia senil», en Revista JANO, núms. 17-23.

- (1991): Enfermedad de Alzheimer, Barcelona: Prous.

CASAlS, I. (1982): Sociología de la ancianidad, Madrid: Mezquita.

COPERIAS, E. (1993): "Cuando la memoria falla», en Muy Interesante, diciembre.

Colmenarejo, C., et al. (1989): «Prevalencia de demencia en el área sur de Madrid. Resultado preliminar de un estudio piloto", en Neurología.

Del Campo, S., y Navarro, M. (1985): Análisis sociológico de la familia española, Barcelona: Ariel. 
Domínguez, C. (1991): "Consecuencias sociales del envejecimiento», en Revista JANO, núm. XL.

Dumont, G. F. (1992): Demographie. Analyse des populations et demographie economique, París: Dunod.

Estudios Sociológicos B. KRIEF (1987): El médico y la tercera edad, Madrid: Laboratorios Beechman.

Fanchamps, A. (1991): Deterioro mental relacionado con la edad y demencias, Barcelona: Ed. Mayo.

Flaquer, L., y Soler, J. (1990): Permanencia y cambio en la familia española, Madrid: CIS.

FrIES, J. (1980): "Aging, natural death, and the compression of morbidity", en New England Journal of Medecine, 303.

- (1983): "An introduction to the compression of morbidity», en Gerontológica Perspecta, 1.

Hugonot, R. y L. (1988): Atlas du Vieillissement et de la Vieillesse, Toulouse: Ed. Erès.

INSA, R., y VERDÚ, L., et al. (1989): «Prevalencia de la demencia en la edad geriátrica», en Actas del III Congreso Internacional de Farmacoterapia y Nutrición Geriátrica, Alicante.

Instituto Nacional de Estadística (1993): Censo de la Población de España de 1991, Madrid: INE.

JANO. Monografías Médicas (1989): Demencia Senil, Barcelona: Doyma, vol. 3, núms. 4 y 5.

Jorm, A. F. (1990): The epidemiology of Alzheimer's disease and related disorders, Londres: Chapman \& Hall.

Journal of Aging and Health (1991): "Living Longer and Doing Worse?...», número extraordinario, vol. 3, núm. 2, mayo.

Katzman, R., et al. (1988): "Advances in the diagnosis of dementia», en R. Terry (ed.), Aging and the Brain, Nueva York: Raven Press, vol. 32.

LABORATORI DE SOCIOlOgiA DE L'ICESB (1992): La demència senil a Barcelona, Barcelona: Ajuntament de Barcelona.

Lay, C., y Woods, B. (1984): Caring for the person with dementia. A guide for families and others carers, Londres: Alzheimer Disease Society.

Lово, A., et al. (1989): "Las demencias en la ciudad de Zaragoza: datos preliminares de un estudio epidemiológico", en $R A E N$.

Mace, N. L., y Rabins, P. V. (1988): Cuando el día tiene 36 horas, México: Pax México.

Mata, M., et al. (1980): "Epidemiología de las demencias», en A. Portera y F. Bermejo, Demencias, Madrid: Magoria.

MCKhan, G., et al. (1984): "Clinical Diagnosis of Alzheimer's Disease», en Neurology.

Moragas, R. (1991): Gerontología soial. Envejecimiento y calidad de vida, Barcelona: Herder.

Mullan, M. Garwford (1993): "Genetic and Molecular advances», en Alzheimer's Disease, TINS, vol. 16, núm. 10.

OCDE (1988): Le vieillessement démographique: conséquences pour la politique sociale, París: OCDE.

OMS (1987): Les tendances démographiques dan la Region européenne, Copenhague: OMS, Bureau Régional de l'Europe.

Olshansky, S.; Carnes, B., y Cassel, C. (1990): «In Search of Mathuselah: Estimating the Upper Limits to Human Longevity", en Science, vol. 250.

- (1993): «Envejecimiento de la especie humana», en Investigación y Ciencia, núm. 201, junio.

OlshansKY, S., et al. (1991): "Trading Off Longer Life for Worsening Health: The Expansion of Morbidity Hipothesis», en Journal of Aging and Health, vol. 3, núm. 2.

Padierna, J., y MARTÍnez, E. (1988): «Demencia y carga familiar», en Revista Psiquis, vol. IX.

Pelletier, K. (1986): Longevidad. Cómo alcanzar nuestro potencial biológico, Barcelona: E. Hispano Europea.

PÉreZ, M. (1991): Familiares y cuidadores ante los enfermos de Alzheimer, Alicante: Fundación Alicante Pro-Vida.

Portera, A., y Bermejo, F. (coords.) (1980): Demencias, Madrid: Magoria.

PuYOL, R., et al. (1993): Los grandes problemas actuales de la población, Madrid: Ed. Síntesis.

RoccA, W. H. (1993): «Epidemiología de la enfermedad de Alzheimer: una actualización», en Demencias. Conceptos actuales, Madrid: Díaz de Santos. 
SÁncheZ Vera, P. (ed.) (1993): Sociedad y población anciana, Murcia: Universidad de Murcia.

SElKoe, D. (1992): Proteina amiloidea y enfermedad de Alzheimer, Barcelona: Fontalba.

Selmes, J., y Selmes, M. (1990): Vivir con la enfermedad de Alzheimer, Madrid: Meditor.

SPiegel, R. (1987): «Demencia senil: definición, terminología, clasificación», en Revista Clínica Española, vol. 181.

Tolosa, E. (1990): Enfermedad de Alzheimer, Barcelona: Doyma.

Thomas, P., et al. (1990): ABC de Enfermedad de Alzheimer, París: Masson.

VV.AA. (1991): Symposium Bases Neuroquímicas del Envejecimiento Cerebral: Alzheimer, Granada: Universidad de Granada.

- (1992): Ya las ideas se pierden...: enfermedad de Alzheimer, Bilbao: Asociación de Familiares de Alzheimer.

Woods, R. (1992): La enfermedad de Alzheimer, Madrid: Instituto Nacional de Servicios Sociales.

Wurtman, R. J. (1991): «Enfermedad de Alzheimer», en Libros de Investigación y Ciencia, Barcelona.

\section{RESUMEN}

El envejecimiento biológico y el envejecimiento demográfico son dos procesos profundamente relacionados entre sí. El notable incremento, absoluto y relativo, de las personas mayores de 65 años, y sobre todo el de las de más de 75 años, es una de las características del nuevo régimen demográfico al que están asistiendo los países desarrollados y es también, en alguna medida, un triunfo de la humanidad en su lucha contra la enfermedad y la muerte. Afortunadamente, la tradicional asociación de vejez con enfermedad, pobreza, soledad o estados carenciales en general está empezando a desaparecer gracias a las mejores condiciones de vida de las personas mayores. Sin embargo, existen enfermedades que, aunque no sean consecuencia de la vejez, aparecen con mayor frecuencia a edades avanzadas, con lo que su presencia entre la población anciana aumenta a medida que ésta también lo hace. Una de ellas es la enfermedad de Alzheimer. El presente artículo expone parte de un estudio más amplio, realizado en la provincia de Alicante, sobre el presente y el futuro de la incidencia y prevalencia de esta enfermedad, con la intención de manifestar que estamos ante un problema no sólo médico o sanitario, sino también familiar y social.

\section{ABSTRACT}

Individual and population ageing are two extremely related processes. The important increase in the proportion and in the actual number of the old population (65 years and over) and the old-old population ( 75 years and over) is one of the characteristics of the new demographic regime in developed countries. Fortunately, the traditional association between old age and disease, poverty or deficiencies in general, is now disappearing. Old people have made enormous progress in their living conditions, in recent years. Nevertheless, there are some diseases, not necessarily consequent of oldness, which appear more frequently in old age. These type of diseases can increase in proportion as the number of elderly increases too. One of these is Alzheimer's disease. The present article presents the main findings of a broader study about the incidence and prevalence of Alzheimer's disease in Alicante. We conclude that Alzheimer' disease is a medical problem as well as a familiar and social one. 